\title{
Personal Experiences with Diagnostic Delay Among Axial Spondyloarthritis Patients: A Qualitative Study
}

\author{
Catherine E. Dube (D) - Kate L. Lapane (D) - Katarina A. Ferrucci · \\ Ariel L. Beccia · Sara K. Khan · Esther Yi · Jonathan Kay (D) · \\ Kristine A. Kuhn · Alexis Ogdie · Shao-Hsien Liu (D)
}

Received: April 9, 2021 / Accepted: May 12, 2021 / Published online: May 31, 2021

(C) The Author(s) 2021

\begin{abstract}
Introduction: On average, patients with axial spondyloarthritis (axSpA) suffer from symptoms up to 13 or more years before diagnosis, contributing to psychological distress and healthcare burden

Methods: We conducted six semi-structured focus groups with 26 axSpA patients (from 3 rheumatology practices located in the states of
\end{abstract}

Supplementary Information The online version contains supplementary material available at https:// doi.org/10.1007/s40744-021-00321-z.

C. E. Dube $(\bowtie) \cdot$ K. L. Lapane · K. A. Ferrucci ·

A. L. Beccia · S. K. Khan · J. Kay - S.-H. Liu

Division of Epidemiology, Department of

Population and Quantitative Health Sciences,

University of Massachusetts Medical School, 368

Plantation Street, Worcester, MA 01655, USA

e-mail: catherine.dube@umassmed.edu

K. A. Ferrucci - A. L. Beccia

Clinical and Population Health Research Program, Graduate School of Biomedical Sciences, University of Massachusetts Medical School, Worcester, MA, USA

E. Yi

Novartis Pharmaceuticals Corporation, East

Hanover, NJ, USA

J. Kay · S.-H. Liu

Division of Rheumatology, Department of

Medicine, UMass Memorial Medical Center,

Worcester, MA, USA
Massachusetts, Colorado, and Pensylvania, USA) exploring early disease and diagnostic experiences. Verbatim transcripts were coded using a start list with emerging thematic codes added. A qualitative thematic analysis was performed

Results: Many participants described meandering and frustrating diagnostic journeys. Participants reported that intermittent axSpA symptoms and idiopathic pain contributed to physician confusion and delay in patients seeking care. Participants were sometimes perceived as somaticizing, drug-seeking, or "crazy." Diagnostic delay led to frustration and mental

\section{K. A. Kuhn}

Division of Rheumatology, Department of Medicine, University of Colorado School of Medicine, Aurora, CO, USA
A. Ogdie
Epidemiology and Biostatistics, Center for Clinical Epidemiology and Biostatistics, University of Pennsylvania Perelman School of Medicine, Philadelphia, PA, USA
A. Ogdie
Department of Rheumatology, University of Pennsylvania Perelman School of Medicine, Philadelphia, PA, USA 
suffering. Doctors "giving up" was considered profoundly negative. Stories of symptoms fell into five areas: (1) pain; (2) stiffness; (3) impact on sleep; (4) impact on daily activities; and (5) changes with weather. Self-advocacy and family advocacy were considered essential. Participants suggested wider use of HLA-B27 testing and development of a definitive diagnostic test

Conclusion: Most participants described significant suffering prior to axSpA diagnosis which could have been avoided with earlier intervention. Further research on the early disease experiences of axSpA patients is needed.

Keywords: Axial spondyloarthritis; AxSpA; Diagnostic delay; Symptoms of unknown origin; Clinical inertia

\section{Key Summary Points}

\section{Why carry out this study?}

The prevalence of axial spondyloarthritis ( $\operatorname{axSpA})$ is estimated to be between 0.35 and $1.4 \%$ in the USA, similar to that of rheumatoid arthritis (0.5-1.03\%).

On average, patients with axSpA suffer from symptoms up to 13 years or more before diagnosis, contributing to psychological distress and healthcare burden.

Only one smaller qualitative study of patient voices and experiences with axSpA diagnostic journeys exists.

\section{What did the study ask?}

What are the early disease stories and experiences of patients with axSpA prior to diagnosis?

\section{What was learned from the study?}

Patients with axSpA with long diagnostic delays reported becoming frustrated and demoralized. They often felt unheard and misunderstood by clinicians while they experienced frequent, sometimes debilitating pain that significantly impacted their daily lives. More research is needed to elicit early disease experiences of newly diagnosed axSpA patients.

Self-advocacy and family advocacy in support of patients were seen as essential; improved awareness of axSpA and improved physician training are needed

\section{DIGITAL FEATURES}

This article is published with digital features, including a summary slide, to facilitate understanding of the article. To view digital features for this article go to https://doi.org/10.6084/ m9.figshare.14566266.

\section{INTRODUCTION}

The prevalence of axial spondyloarthritis (axSpA) is estimated to be between 0.35 and $1.4 \%$ in the USA $[1,2]$, similar to that of rheumatoid arthritis (0.5-1.03\%). [2] In the USA, for patients satisfying classification criteria for $\operatorname{axSpA}$, the average time to diagnosis has been reported to be up to 13 years or longer [3]. Diagnostic delay has been associated with worse clinical outcomes, including greater disease activity, poorer physical function, and more structural damage [4]. At onset, patients with axSpA are typically young (average age of onset ranges from 25 to 28 years) [5], otherwise healthy, and do not appear sick [6]. Most patients experience back pain as their initial symptom [7], an otherwise common complaint in primary care practices (lifetime prevalence $60-70 \%)$. The majority of back pain is mechanical in nature and is, for the most part, neither progressive nor permanent [8]. 
Inflammatory back pain (IBP), a distinguishing form of back pain in axSpA, is characterized by an insidious onset, duration of pain of $>3$ months, improvement of pain with activity, pain at night with improvement upon waking, and no improvement with rest. An estimated $5-6 \%$ of patients with chronic back pain in the USA have IBP [9-12]. Physicians caring for patients early in the course of axSpA may find it difficult to differentiate this condition from common mechanical back pain. Together, these factors delay appropriate diagnostic testing and referrals to rheumatologists [5].

Undiagnosed axSpA patients suffer psychologically, experiencing distress, depression, and/or desperation during their prolonged search for diagnosis and treatment [4, 13]. Early treatment of axSpA has been associated with improved disease management, reduced likelihood of disease progression, improved longterm patient well-being, and reduced healthcare costs $[4,14]$.

To learn about the experiences of patients with axSpA, we conducted exploratory semistructured focus groups in March-June 2019. Focus groups were designed to generate indepth discussion around early disease and diagnostic experiences. We aimed to explore relevant, overt, and latent issues to improve understanding from the perspective of patients. This study was part of the SpondyloArthritis Screening and Early Detection (SpA-SED) study [15].

\section{METHODS}

A qualitative approach employing open-ended, semi-structured focus group discussions was employed. Focus group methods have been previously described [16], and our focus group protocol (see Electronic Supplementary Material [ESM] ) was guided by best practices for the conduct and reporting of qualitative research (COREQ) [17]. Ethical approval was obtained via the University of Massachusetts Medical School Institutional Review Board (IRB). A reliance agreement was provided by the University of Pennsylvania IRB. The authors guarantee that certification of the study was performed in accordance with the ethical standards as laid down in the 1964 Declaration of Helsinki and its later amendments or comparable ethical standards.

Eligible focus group participants met the following inclusion criteria: (1) had a diagnosis of axSpA confirmed by a rheumatologist; (2) had capacity to consent; and (3) were aged $\geq$ 18 years. Excluded were individuals who refused to participate in the focus group discussions or audio recording or who were not Englishspeaking. Participants were invited using a purposive approach via three rheumatology practices (Massachusetts, Colorado, and Pennsylvania) following our recruitment protocol (ESM 1). One individual was no longer interested and withdrew after recruitment and is not included in participant totals. Authors $\mathrm{CD}, \mathrm{AB}$, and $\mathrm{KF}$ had contact with participants during recruitment and scheduling. Interested eligible participants were scheduled for a 2 -h focus group conducted in a hotel meeting room in their local area (Table 1).

\section{Approach to Focus Groups}

The author CD (an experienced qualitative researcher who teaches a graduate course in qualitative methods) conducted the focus groups following a semi-structured framework guided by a pilot-tested focus group protocol. $\mathrm{CD}$ introduced herself and her professional role at the beginning of the focus group. One research assistant was present and collected observational notes. No one else attended. Discussions were recorded using dual audio recorders. Elements of consent were reviewed per the IRB protocol using a Fact Sheet (ESM) prior to the discussion. Focus group questions are included in ESM 2. A semi-structured, openended approach was employed. The intent of the focus group discussion was to elicit and capture the experiences of patients with axSpA with diagnostic journeys and diagnostic delays in their own words. Data collected was comprised of participant comments, thoughts, and utterances from their own perspective emanating from discussions that were gently guided by 
Table 1 Focus group composition and duration of substantive discussion

\begin{tabular}{lllllll}
\hline FG group features & FG1 & FG2 & FG3 & FG4 & FG5 & FG6 \\
\hline Location $^{\mathrm{a}}$ & Worcester & Worcester & Aurora & Aurora & Worcester & Philadelphia \\
Number of participants & 7 & 4 & 5 & 3 & 4 & 3 \\
Duration & $1 \mathrm{~h} 48 \mathrm{~min}$ & $1 \mathrm{~h} 20 \mathrm{~min}$ & $1 \mathrm{~h} 53 \mathrm{~min}$ & $2 \mathrm{~h} 8 \mathrm{~min}$ & $2 \mathrm{~h}$ & $1 \mathrm{~h} 41 \mathrm{~min}$ \\
\hline
\end{tabular}

$F G$ Focal group

a Worcester MA; Aurora CO; Philadelphia PA, USA

the moderator to remain on topic. Specific data (like number, type, and training of physicians being discussed) were not specifically elicited as this would interfere with the natural flow and content of the focus group discussion. After each focus group was completed, additional demographic data were collected. Participants were provided with light refreshments and $\$ 100$ gift/cash card to compensate for time and travel expenses. No repeat interviews were conducted for this focus group study.

Audio files were uploaded to a secure research drive housed at the University of Massachusetts Medical School (UMMS) and then the original audio files residing on portable recording devices were deleted. We employed a HIPAA (U.S. Health Insurance Portability and Accountability Act of 1996; https://www.hhs.gov/hipaa/index.html) compliant transcription service for verbatim transcription. Completed transcripts were reviewed and cleaned by research assistants. Transcripts were de-identified by replacing participant names with ID numbers.

Additional textual data were volunteered by some participants (e.g., written reflections, a diary of disease experience, follow-up emails to author CD) and were de-identified for use as source documents. Observer notes and reflections from team members were also transcribed. Transcripts were not returned to the participants for review. De-identified transcripts and other source documents were uploaded to NVivo for coding (QSR NVivo; QSR international Pty ltd., Melbourne, Australia, 2000; https://www.qsrinternational.com/nvivo/ nvivo-products).

\section{Analytic Approach}

A thematic analysis was conducted using a coding start-list. The start-list was derived from the focus group protocol questions (ESM 2), and new codes were added as they were identified during coding.

All transcripts were dual coded for accuracy by two researchers (KF, SK) reaching intercoder reliability $>95 \%$ agreement. Coding was merged and reports were created and reviewed. Ten codes most relevant for further analysis were identified: Advice4MD; Done Differently; Diagnosis; Early Symptoms; Helped Dx; Impede Dx; Pain Complain; Stiffness; Told MD; and Wrong Dx (ESM 3). For each priority code, two summaries of impressions and key quotations were independently prepared by two researchers. In the case of "Diagnosis," the lengthy code report was recoded into four component subcodes (Delay; Feelings; Getting Dx; Search for Diagnosis) and subcode summaries were prepared. Impressions and consensus compilations were created based on review and discussion of each code and subcode summary and original code reports. Source documents generating the findings below included summaries, impressions, compilations, and code reports. Draft findings were reviewed by the research team, and the results reported below were agreed upon. Focus group participants were provided a summary of findings, and feedback was requested although none was returned. 
Table 2 Focus group participants with axial spondyloarthritis-characteristics

\begin{tabular}{|c|c|}
\hline Demographics & Values \\
\hline Age at participation in focus group (years) & $\begin{array}{l}53.5 \\
\quad(21-76)\end{array}$ \\
\hline Age at appearance of first symptoms (years) & $\begin{array}{l}22.8 \\
\quad(10-55)\end{array}$ \\
\hline Age at diagnosis (years) & $\begin{array}{l}34.8 \\
(15-65)\end{array}$ \\
\hline $\begin{array}{l}\text { Years from appearance of first symptoms to } \\
\text { diagnosis }\end{array}$ & $12(1-37)$ \\
\hline $\begin{array}{l}\text { Years from first complaint to a doctor until } \\
\text { diagnosis }\end{array}$ & $10(0-30)$ \\
\hline \multicolumn{2}{|l|}{ Gender, $n$} \\
\hline Man & 16 \\
\hline Woman & 10 \\
\hline \multicolumn{2}{|l|}{ Education, $n$} \\
\hline High School or Less & 0 \\
\hline Some College & 10 \\
\hline College Graduate & 8 \\
\hline Graduate Degree & 8 \\
\hline \multicolumn{2}{|l|}{ Race/ethnicity, $n$} \\
\hline Non-Hispanic White & 20 \\
\hline Black, African American & 2 \\
\hline Asian & 2 \\
\hline Hispanic & 1 \\
\hline Other & 1 \\
\hline
\end{tabular}

Values are presented as the mean with the range in parenthesis or as a frequency (number), as appropriate

\section{RESULTS}

We conducted six focus groups averaging approximately four participants per group (26 participants in total) and ranging in duration from $1 \mathrm{~h}$ and $20 \mathrm{~min}$ to $2 \mathrm{~h}$ and $8 \mathrm{~min}$ (Table 1 ). The mean participant age was 53.5 years, and the majority were men (61.5\%) and non-
Hispanic White (77\%). All participants had attended a college and $31 \%$ had graduate degrees. The average diagnostic delay was 12 years (appearance of first symptoms to diagnosis) (Table 2). Data saturation was attained as no new concepts were revealed in the final focus groups. With saturation, the sample size we achieved was determined to be sufficient.

Table 3 shows themes and representative quotes of the participants' experiences organized across five topic areas: 1 -experiences with clinicians; 2-symptoms of early disease; 3-common misdiagnoses; 4-self-advocacy; 5-human leukocyte antigen B27 (HLA-B27). In Table 3, participant quotes are identified by gender and age, and themes are italicized. Due to word limitations divergent cases are not provided.

\section{Experiences with Clinicians}

Many participants described long, circuitous diagnostic journeys. These journeys involved multiple clinicians, including primary care and specialist physicians, physical therapists, and chiropractors; however, most explicit comments about participant experiences focused on physicians. Some physicians used a trial-anderror approach to see if treatment with various medications might help in the absence of a clear diagnosis. Many participants described a variety of difficult experiences with doctors, ranging from minimizing or dismissing pain complaints to concluding that symptoms were "psychosomatic" or imaginary. Some physicians seemed to imply that the participant was trying to obtain narcotics or was "crazy."

With a slowly progressing disease with symptoms that have no easily identifiable source patients strove to impress upon clinicians that something is genuinely wrong. Thus patients waited for a serious flare-up or used dramatic language to convey their distress (e.g., using a 1-10 pain scale, calling 10 "suicidal" [male aged 46]). Some participants reported an under-appreciation by physicians for symptoms that would come and go. These intermittent symptoms appeared to confuse some of their physicians and caused some patients to delay seeking care. 


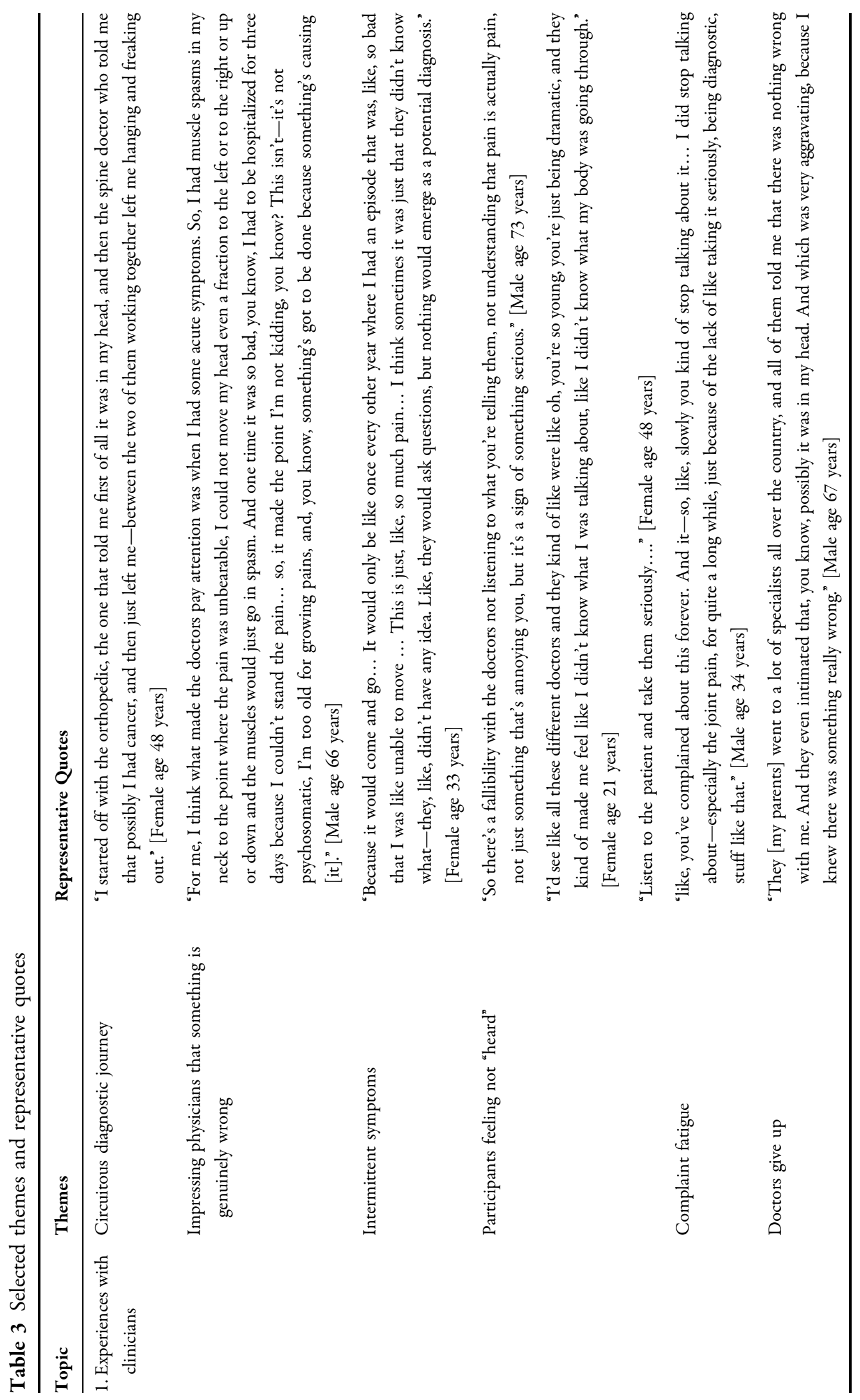




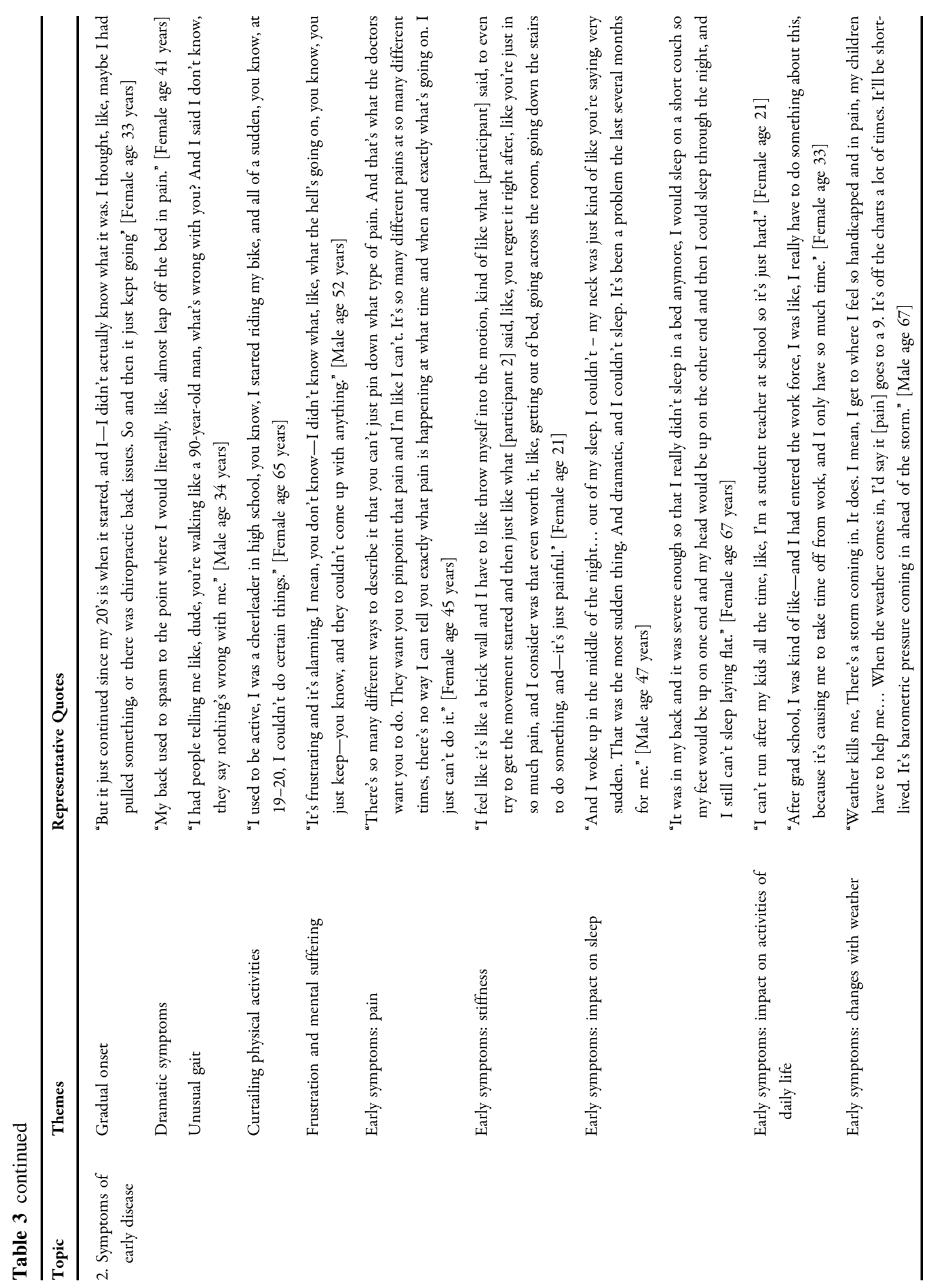




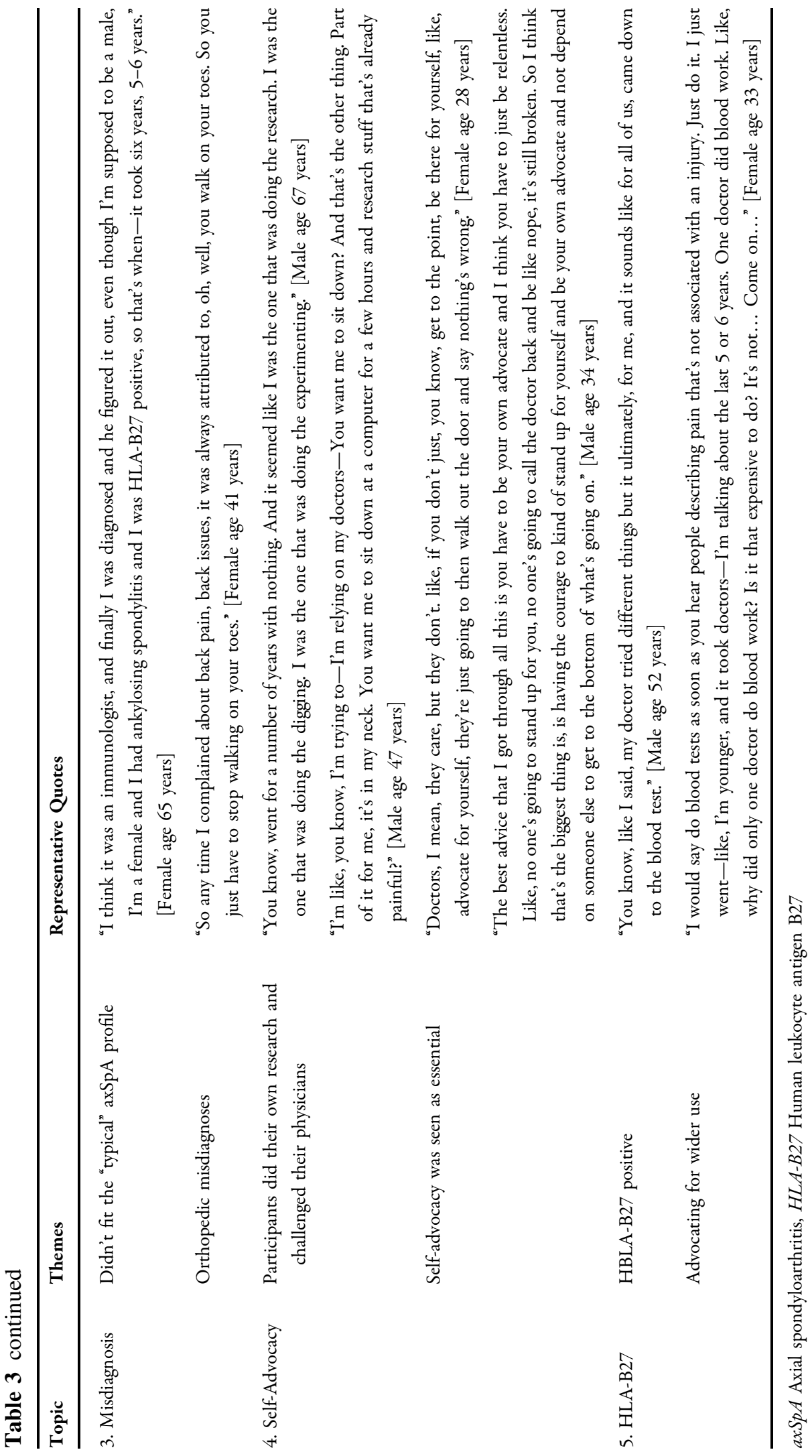


Participants repeatedly reported feeling neither "heard" nor believed. Their advice to physicians was straight-forward: allow patients to explain their symptoms, hear them, and believe them. Participants desired clinicians who were present and persistent, and who followed through to find an answer. Some participants described a fatigue after trying unsuccessfully to express concern about their symptoms or receive treatment. When doctors gave up on a patient's diagnostic quest, it was experienced as profoundly negative.

\section{Symptoms of Early Disease}

Participants described a variety of early symptoms, primarily pain, that caused them to seek medical care. For some the onset of symptoms was gradual (a "pulled muscle" or what was assumed to be a sports injury that never got better). For others the initial onset was more dramatic ("I would just wake up at night screaming in pain." [Female age 41 years]). Back pain was a common presenting symptom. Other early pain locations included buttocks, neck, knees, legs, ankles, feet, hands, chest/ sternum, clavicle, "weird shoulder pain," sciatic pain, and migratory pain. Pain was described as shooting, stabbing, aching, soreness, constant or "on and off," and ranged in intensity from relatively minor to so bad that the participant was "in tears." One participant reported, "the doctor told me I had strange aches and pains." [Female age 65 years]. With younger patients, clinicians sometimes attributed pains to "growing pains." Severe pain was described as "in so much pain I couldn't move" or "When I find that I breathe in, I feel like I have icepicks sticking through my ribs." [Male age 53 years]. Some participants experienced pain after remaining in one position for a long time. Others experienced very painful spasms in their neck, back, or hips.

Participants also described a noticeable $u n u$ sual gait or "funny gait," or a "bending over" posture. Many participants described a need to curtail normal physical activities or sports. Other early symptoms included an inability to lift their leg (e.g., to get out of the car), pain at night disturbing sleep, an inability to sleep flat or sleep in a bed, morning stiffness relieved after long shower, and severe eye pain.

Participants discussed early disease experiences as they started seeking answers and relief from their doctors. Early symptom experiences were varied, and some patterns of symptoms made estimating duration of symptoms difficult. Many participants experienced frustration and mental suffering during the journey toward a diagnosis.

Table 3 shows representative quotes for early symptom stories organized into five themes: (1) pain; (2) stiffness; (3) sleep; (4) daily activities; and (5) changes with weather. With respect to pain (frequency, timing, intermittent patterns), some participants had constant pain that flared up, whereas others had short bouts of pain that subsided and re-emerged. For some, pain was localized in one or only a few locations, while others experienced waxing and waning pain that migrated around their body, making it difficult to describe location or duration. Participants described pain intensity either by its life impact or by using a numeric pain scale. The numeric scale had limitations, as some patients reported baseline pain levels above 5 on the scale, and other patients describe their pain at times as "off the charts." Stiffness was described in a variety of ways, mostly by which activities it prevented participants from performing-like getting out of bed or cutting their toenails. They also described strategies to deal with stiffness by "breaking through" the stiffness regardless of pain. Impact on sleep was mentioned by some as among their earliest complaints ("[waking] up at night screaming in pain" [female age 41 years]) and later in disease progression when it was impossible to find a comfortable position for sleep. Impacts on sleep were some of the most upsetting issues for participants. Participants described how symptoms affected their ability to continue daily activities, including work, driving, caring for children, shopping, personal hygiene, and sports or other recreational activities. Many but not all participants described dramatic changes in symptoms concurrent with changes in weather, particularly with an approaching storm. Some participants attributed flare-ups to changes in barometric 
pressure, or to increased humidity. Two participants also mentioned worsening of their symptoms precipitated by a change in altitude (i.e., returning to a high-altitude location).

\section{Misdiagnosis}

Some participants, especially women, reported misdiagnosis because they didn't fit the "typical" axSpA profile (i.e., male, predominantly axial skeletal involvement, aged in their 30's). This resulted in further diagnostic delay and frustration. Several participants had been told that their symptoms were due to growing pains or the result of sports injuries. The sports injury most commonly implicated was a "pulled muscle." Other presumed sports injuries included a "sprained ligament," "bruising of the bone," dehydration, and "chronic groin pull." A few participants reported that their doctor had attributed their symptoms to one leg being shorter than the other and advised them to wear a wedge in their shoe. Other orthopedic misdiagnoses included "flat feet," "toe walking," frozen shoulder, and back problems such as a bulging disk, "slipped disk," "pinched nerve," or sciatica. Participants mentioned a variety of different rheumatologic misdiagnoses, including rheumatoid arthritis, juvenile rheumatoid arthritis, psoriatic arthritis, "some form of arthritis," or a "mild case of arthritis." One participant was appropriately diagnosed with inflammation of the sacroiliac joints-with the caveat "but we don't know why." Other misdiagnoses included osteomyelitis, Duchenne muscular dystrophy, cancer, and a sleep disorder.

Several participants had multiple misdiagnoses. "They would do tests on me all the time and it was always something new [new disease]... It took a few years before it came up, the AS." [Male age 46 years]. Other participants had no diagnosis for a prolonged period of time: "They would ask questions, but nothing would emerge as a potential diagnosis." [Female age 33 years]. To help participants cope with symptoms, physicians sometimes advised patients to change their jobs or career, reduce their activities ("You're doing too much"), go to a physical therapist or chiropractor, or simply warm-up before exercising.

\section{Self-Advocacy}

All participants ultimately received a verified diagnosis of axSpA. For many, this was due to their own and/or their family's tenacity to find answers. Some participants had the confidence to challenge their physicians about their initial diagnosis. During their prolonged diagnostic journey, most participants felt widely misunderstood by physicians. Advocacy was seen by most participants as essential to advancing the diagnostic process often over years. Some resorted to their own research and self-diagnosis, often confirmed when they convinced a doctor to obtain HLA-B27 testing or a rheumatology referral. This level of advocacy was viewed as challenging but necessary to sustain over time. Some patients resented having to do their own research, and self-advocacy was considered particularly challenging when patients were sick.

\section{Human leukocyte antigen B27}

Most participants were $H L A-B 27$ [18] positive. Although many understood that this test was not by itself diagnostic for axSpA, it often was a key factor in their receiving a definitive diagnosis. Some reported having other family members who also carried the gene, some of whom were asymptomatic. A few participants were unsure if they had been tested for HLAB27. None reported a negative test. In general, participants would have preferred to have been tested for the HLA-B27 antigen earlier. Some participants advocated for wider use of HLA-B27. One participant suggested adding HLA-B27 to routine "arthritis panels". Participants believed that early administration of a definitive diagnostic test for axSpA would have alleviated both their physical and emotional suffering. One participant explicitly stated the need to develop a definitive diagnostic test for axSpA. 


\section{DISCUSSION}

In its early stages, axSpA can be confusing and distressing for patients given the variety of clinical manifestations with which may present [19]. The onset of back pain in younger individuals may be attributed to mechanical factors, such as injury or overuse, rather than to the initial presentation of axSpA. Early in the disease course, symptoms may not be accompanied by radiographic confirmation as the disease may take years to develop to the stage at which this is possible [20]. This makes it challenging for clinicians who must rely on clinical experience and expertise [20] which many may not possess. These factors contribute to the potential for a long delay in diagnosis.

Some participants discussed HLA-B27 testing and supported its wider use in screening for and diagnosis of axSpA. When the HLA-B27 antigen is present in an individual with back pain of at least 3 months duration, the presence of two additional clinical features of spondyloarthritis would satisfy both the Amor classification criteria for spondylarthropathy [21] and the Assessment of SpondyloArthritis international Society (ASAS) classification criteria for axSpA [22]. If healthcare providers were familiar with these criteria, HLA-B27 testing would facilitate early diagnosis of axSpA.

Few participants commented on the role of magnetic resonance imaging (MRI). Bone marrow edema, indicating the presence of osteitis, may be detected by MRI in sacroiliac joints, several years before sacroiliitis is evident on plain radiographs [23]. Additionally, patients with inflammatory back pain but without evidence of sacroiliitis on plain radiographs may display evidence of osteitis on MRI scans, consistent with the diagnosis of non-radiographic axSpA [24]. Thus, earlier and broader use of this technology to assess individuals with inflammatory back pain might hasten the diagnosis of axSpA.

Delay in axSpA diagnosis is a problem in the USA and around the world. In 2019, Yi et al. conducted a systematic review [4] in which 1391 English language publications were retrieved and 21 original, non-interventional studies published between 2009 and 2018 were included for analysis. In these studies, conducted in 13 countries (but none in the USA), mean diagnostic delay ranged from approximately 4 to 12 years. In the USA, estimated delay in diagnosis is as long as 14 years [5]. Our focus group participants' mean time from appearance of first symptoms to diagnosis was 12 years, falling into the upper range of both of these estimates.

All our focus group participants had a validated diagnosis of axSpA. They described their often lengthy series of healthcare experiences as they searched for a diagnosis and symptom relief. In many cases, overwhelming, life-affecting pain and physical limitations caused serious concern and suffering. The period when participants remained undiagnosed was most disturbing to them, especially for those who persisted as they struggled to find a clinician who believed their story. Many participants described how they became their own advocates, at times pushing doctors to hear and respond to their requests for answers, further testing, symptom relief, treatment, and/or referral. Several participants related stories about seeking help and advice from multiple doctors, both near and far, finally settling on a doctor who could meet their diagnostic, treatment, and informational needs while providing supportive care.

Patients switching from doctor to doctor, or "doctor shopping," may triggger a negative reaction among healthcare providers. "Doctor shopping" is a pejorative term often used in the context of drug-seeking patients. Indeed, several of our axSpA participants lamented doctors treating them as if they were seeking narcotics when they were not. Predictably, focus group participants regarded their search for a doctor as an honest quest for care stemming from desperation after repeated failures with those healthcare providers who could not offer a diagnosis, relief or, in a few cases, empathy or sympathy.

Patients are likely not fully aware of the many barriers affecting axSpA diagnostic delay in the USA [15], and some dynamics affecting this delay may not be apparent to clinicians either. Forces such as clinical inertia may 
impede providers' ability to initiate diagnostic procedures or effective treatments [25]. Clinical inertia is commonly encountered in conditions where symptoms are present but the underlying cause is yet to be discovered. Provider perceptions and behaviors contributing to clinical inertia may include: (1) an overestimate of the care provided; (2) therapeutic avoidance and a rationale using "soft" evidence and a belief that current care is adequate; and (3) training and organizational deficiencies. [25] In the case of axSpA, limited visit time and fluid symptoms may also have an impact. Patient-related factors that may contribute to clinical inertia include poor communication skills and low health literacy [26]. To address clinical inertia in general, the adoption of person-centered care practices and improved clinician education have been recommended $[26,27]$ and for axSpA specifically, increased awareness among non-rheumatologist healthcare providers caring for backpain is suggested $[5,28]$. These potential improvements-person-centered care, better communication, more clinician education, and increased awareness of axSpA-were all endorsed by various focus group participants in our study.

AxSpA patient experiences as described in the focus groups (e.g., having multiple vague and changing symptoms, lack of objective clinical findings in early disease, having to resort to dramatic language to describe severe symptoms, developing feelings of frustration and helplessness, being forced into a position where patients may have to actively advocate with healthcare providers to get adequate care) illustrate the difficulty and frustration that these individuals experience. Making matters worse, physicians may treat people with undiagnosed axSpA as "difficult patients" [29-32]. Often under stress with multiple vague or exaggerated chronic symptoms, "difficult patients" [33] have been described as frequent consumers of healthcare services and may be perceived as "angry, defensive or frightened" [34]. Such patients may evoke negative feelings and aversive reactions from physicians, resulting in compromised medical care [35] and feelings of distress or abandonment among patients. In our study, participants highly valued those clinicians who understood and believed them, took them seriously, and did not give up before a diagnosis was made, even though it may have taken a long time. These participants found comfort in these trusted physicians and described deep appreciation for their efforts.

To our knowledge, only one other smaller qualitative study of patient voices and experience with axSpA diagnostic journeys exits, conducted in the UK [36]. Among quantitative studies, in a recent survey Ogdie et al. collected self-reported survey data on the experiences of patients with ankylosing spondylitis during their diagnostic journey [37]. However, while these authors addressed some of the same issues as our study, they used a vastly different methodological approach [37]. Our study presents a unique perspective due to its qualitative nature. Qualitative techniques are designed to provide a unique depth of understanding which cannot be reproduced in structured survey form. Unlike surveys, qualitative research encourages the free disclosure of feelings, ideas, opinions, and experiences without constraint while facilitating the discovery of personal and potentially unexpected findings that emerge organically without direct questioning. The use of qualitative methods in the present study allowed an in-depth exploration of early disease and diagnostic experiences among patients with axSpA in the USA and therefore addressed an important knowledge gap.

\section{Limitations}

Our findings should be interpreted with limitations in mind. The majority of participants in our focus groups were educated, some with graduate degrees. This may have affected patients' persistence and effective self-advocacy. The experience of patients with low health literacy may be different. Participants were also very experienced with axSpA. At the time of the focus group, the average length of time since diagnosis was 18.7 (range 0-51) years. Thus, most participants had knowledge that an undiagnosed or newly diagnosed patient would not yet possess. As with similar qualitative research, 
our sample size was small, limiting generalizability to wider populations.

\section{CONCLUSION}

This is the largest study to date that has used qualitative methods to characterize patient perspectives of the extended journey to a diagnosis of axSpA and the first to include patients from three different geographic regions in the USA. Patients with axSpA described significant suffering which could have been avoided prior to diagnosis and a wide array of initial symptoms. Diagnosis was frequently elusive, even when symptoms restricted normal activities and were sometimes severe. Intermittent symptoms hindered a timely diagnosis and were particularly frustrating for patients. Self-advocacy and family advocacy were seen as essential factors in many patients' successful quest for diagnosis and treatment, an effort that in some cases spanned years. Some participants conducted their own research and self-diagnosis, then challenged their physician to provide referrals or conduct necessary tests. Participants expressed satisfaction with physicians who understood and believed them, took them seriously, and did not give up even with long diagnostic delays. Participants suggested wider use of HLAB27 testing and the development of a definitive diagnostic test. Further study of axSpA patients who are early in their diagnostic journey is needed to determine best practices to support these patients and most effectively reduce diagnostic delay.

\section{ACKNOWLEDGEMENTS}

We thank the focus group participants for sharing their experiences.

Funding. The authors disclose receipt of the following financial support for the research, authorship, and/or publication of this article: Funding for this study and the journal's Rapid Service Fee was provided by Novartis Pharmaceuticals Corporation via a Master Services
Agreement. Research reported in this publication was also supported by a charitable contribution to the UMass Memorial Foundation from Timothy S. and Elaine L. Peterson. Novartis reviewed and provided feedback during the development of the qualitative interview guide, facilitated access to rheumatologists who assisted with participant recruitment, and reimbursed publication costs. The funders had no other role in study design, data collection and analysis, or decision to publish. Dr. Yi of Novartis is a co-author and provided input into preliminary drafts of this manuscript.

Authorship. All named authors meet the International Committee of Medical Journal Editors (ICMJE) criteria for authorship for this article, take responsibility for the integrity of the work as a whole, and have given their approval for this version to be published.

Authors' Contributions. Catherine Dube led the design of this qualitative study and recruitment procedures, moderated all focus groups, led the coding and analysis process, interpreted final results, and drafted the final manuscript. Kate Lapane participated in study design, interpretation of results, and substantially contributed to the final manuscript. Katarina Ferrucci and Ariel Beccia participated in recruitment, assisted in focus groups. Ms. Ferrucci also participated in coding and intermediate qualitative analytical steps with Sara Khan. Jonathan Kay and Esther Yi provided access to resources for recruitment and participated in study design. Jonathan Kay, Kristine Kuhn and Alexis Ogdie participated in study recruitment. Jonathan Kay also provided substantive content for this manuscript. ShaoHsien Liu served as principal investigator, assisted in administrative aspects of the focus group project, and contributed to study design. All authors reviewed and provided critical input into the final manuscript.

Prior Presentation. This manuscript is based on the same work that was also previously accepted for a conference presentation. Parts of this manuscript were presented as a poster at the Annual European Congress of 
Rheumatology (Virtual Conference) EULAR 2021; 2-5 June 2021.

Disclosures. Authors Lapane, Liu, Dube, Beccia, Ferrucci, Kay, Khan, and Kuhn have declared no conflicts. A. Ogdie has declared paid consultation for Abbvie, Amgen, BMS, Celgene, Corrona, Eli Lilly, Gilead, Janssen, Novartis, Pfizer, and UCB and grant funding from Novartis and Pfizer. Her husband has received royalties from Novartis. Dr. Yi is an employee of Novartis.

Compliance with Ethics Guidelines. All procedures in this study were conducted in accordance with the University of Massachusetts Medical School Institutional Review Board (H00019354, H00016873) which granted a waiver of written consent and approved a Fact Sheet (see ESM) for use in the consent process. A reliance agreement was provided by the University of Pennsylvania Institutional Review Board. The authors guarantee that certification of the study was performed in accordance with the ethical standards as laid down in the 1964 Declaration of Helsinki and its later amendments or comparable ethical standards.

In accordance with IRB-approved procedures, the Fact Sheet was reviewed aloud prior to the beginning of the focus group, and participants were given the opportunity for questions or clarifications. Participants were encouraged to leave the focus group without penalty if they had concerns or changed their mind about participation. Participants refusing participation would still receive participant compensation. No participants withdrew from the focus groups. The consent Fact Sheet explained that focus group comments would be recorded, transcribed, de-identified, and used for research purposes. Participants were asked if they would like a copy of subsequent publications. A summary of findings was circulated to participants for their review and feedback prior to manuscript preparation. Quotations from participants are de-identified.

Data Availability. The datasets generated (focus group transcripts) and analyzed during the current study are not publicly available due to contractual agreements with the funder.

Open Access. This article is licensed under a Creative Commons Attribution-NonCommercial 4.0 International License, which permits any non-commercial use, sharing, adaptation, distribution and reproduction in any medium or format, as long as you give appropriate credit to the original author(s) and the source, provide a link to the Creative Commons licence, and indicate if changes were made. The images or other third party material in this article are included in the article's Creative Commons licence, unless indicated otherwise in a credit line to the material. If material is not included in the article's Creative Commons licence and your intended use is not permitted by statutory regulation or exceeds the permitted use, you will need to obtain permission directly from the copyright holder. To view a copy of this licence, visit http:// creativecommons.org/licenses/by-nc/4.0/.

\section{REFERENCES}

1. Strand V, Rao SA, Shillington AC, et al. Prevalence of axial spondyloarthritis in United States rheumatology practices: assessment of SpondyloArthritis International Society criteria versus rheumatology expert clinical diagnosis. Arthritis Care Res (Hoboken). 2013;65:1299-306. https://doi.org/10.1002/ acr.21994.

2. Reveille JD, Witter JP, Weisman MH. Prevalence of axial spondylarthritis in the United States: estimates from a cross-sectional survey. Arthritis Care Res (Hoboken). 2012;64:905-10. https://doi.org/10. 1002/acr.21621.

3. Deodhar A, Mease PJ, Reveille JD, et al. THU0065 prevalence of axial spondyloarthritis among undiagnosed chronic back pain patients in the United States. Ann Rheum Dis. 2014;73:198-9.

4. Yi E, Ahuja A, Rajput T, et al. Clinical, economic, and humanistic burden associated with delayed diagnosis of axial spondyloarthritis: a systematic review. Rheumatol Ther. 2020;7:65-87. https://doi. org/10.1007/s40744-020-00194-8.

5. Danve A, Deodhar A. Axial spondyloarthritis in the USA: diagnostic challenges and missed 
opportunities. Clin Rheumatol. 2019;38:625-34. https://doi.org/10.1007/s10067-018-4397-3.

6. Basile LM. But you don't look sick. 2020. https:// axialspondyloarthritis.net/living/look-sick/. Accessed 25 Jan 2021.

7. Rojas-Vargas M, Munoz-Gomariz E, Escudero A, et al. First signs and symptoms of spondyloarthritis-data from an inception cohort with a disease course of two years or less (REGISPONSEREarly). Rheumatology. 2009;48:404-9. https://academic. oup.com/rheumatology/article/48/4/404/1789827. Accessed 24 May 2021.

8. Lawrence RC, Felson DT, Helmick CG, et al. Estimates of the prevalence of arthritis and other rheumatic conditions in the United States part II. Arthritis Rheum. 2008;58:26-35. https://doi.org/10. 1002/art.23176.

9. Rudwaleit M, Metter A, Listing J, Sieper J, Braun J. Inflammatory back pain in ankylosing spondylitis: a reassessment of the clinical history for application as classification and diagnostic criteria. Arthritis Rheum. 2006;54:569-78. https://doi.org/10.1002/ art.21619.

10. van den Berg R, de Hooge M, Rudwaleit M, et al. ASAS modification of the Berlin algorithm for diagnosing axial spondyloarthritis: results from the SPondyloArthritis Caught Early (SPACE)-cohort and from the Assessment of SpondyloArthritis international Society (ASAS)-cohort. Ann Rheum Dis 2013;72:1646-53. https://ard.bmj.com/content/72/ 10/1646. Accessed 24 May 2021.

11. Weisman MH. Inflammatory back pain: the United States perspective. Rheum Dis Clin North Am 2012;38:501-12. https://doi.org/10.1016/j.rdc. 2012.09.002, https://www.ncbi.nlm.nih.gov/pmc/ articles/PMC3501982/. Accessed 24 May 2021.

12. Sieper J, van der Heijde D, Landewé R, et al. New criteria for inflammatory back pain in patients with chronic back pain: a real patient exercise by experts from the Assessment of SpondyloArthritis international Society (ASAS). Ann Rheum Dis 2009;68: 784-8. https://ard.bmj.com/content/68/6/784. Accessed 24 May 2021.

13. Martindale J. I74. The impact of delay in diagnosing ankylosing spondylitis/axial SpA. Rheumatology. 2014;53(Suppl 1):i16. https://academic.oup.com/ rheumatology/article/53/suppl_1/i16/1794073. Accessed 24 May 2021.

14. Haroon N, Inman RD, Learch TJ, et al. The impact of TNF-inhibitors on radiographic progression in ankylosing spondylitis. Arthritis Rheum. 2013;65: 2645-54. https://doi.org/10.1002/art.38070.
15. Lapane KL, Khan S, Shridharmurthy D, et al. Primary care physician perspectives on barriers to diagnosing axial spondyloarthritis: a qualitative study. BMC Fam Pract. 2020;21:204. https://doi. org/10.1186/s12875-020-01274-y.

16. Lapane KL, Dube C, Ferrucci K et al. Patient perspectives on provider practices leading to an axial spondyloarthritis diagnosis: a qualitative study. BMC Fam Pract. Accepted.

17. Tong A, Sainsbury P, Craig J. Consolidated criteria for reporting qualitative research (COREQ): a 32-item checklist for interviews and focus groups. Int J Qual Health Care. 2007;19:349-57. https:// academic.oup.com/intqhc/article/19/6/349/ 1791966. Accessed 24 May 2021.

18. Sheehan NJ. The ramifications of HLA-B27. J R Soc Med. 2004; 97:10-4. https://www.ncbi.nlm.nih. gov/pmc/articles/PMC1079257/. Accessed 24 May 2021.

19. Malaviya AN, Rawat R, Agrawal N, et al. The nonradiographic axial spondyloarthritis, the radiographic axial spondyloarthritis, and ankylosing spondylitis: the tangled skein of rheumatology. Int J Rheumatol. 2017;2017:1824794. https://www. hindawi.com/journals/ijr/2017/1824794/. Accessed 24 May 2021.

20. Rudwaleit M, van der Heijde D, Khan MA, et al. How to diagnose axial spondyloarthritis early. Ann Rheum Dis. 2004;63:535-43. https://ard.bmj.com/ content/63/5/535. Accessed 24 May 2021.

21. Amor B, Dougados $M$, Mijiyawa $M$. Critères de classification des spondylarthropathies [Criteria of the classification of spondylarthropathies]. Rev Rhum Mal Osteoartic. 1990;57(2):85-9. (In French). https://www.sciencedirect.com/science/article/abs/ pii/S1878622709000034. Accessed 24 May 2021.

22. Rudwaleit M, van der Heijde D, Landewé R, et al. The development of Assessment of SpondyloArthritis International Society classification criteria for axial spondyloarthritis (part II): validation and final selection. Ann Rheum Dis. 2009;68(6): 777-83. https://doi.org/10.1136/ard.2009.108233, https://ard.bmj.com/content/68/6/777.long. Erratum in: Ann Rheum Dis. 2019;78(6):e59. Accessed 24 May 2021.

23. Oostveen J, Prevo R, den Boer J, et al. Early detection of sacroiliitis on magnetic resonance imaging and subsequent development of sacroiliitis on plain radiography. A prospective, longitudinal study. J Rheumatol. 1999;26(9):1953-8.

24. Kiltz U, Baraliakos X, Karakostas P, et al. Do patients with non-radiographic axial spondylarthritis differ from patients with ankylosing spondylitis? Arthritis 
Care Res (Hoboken). 2012;64(9):1415-22. https:// doi.org/10.1002/acr.21688.

25. Phillips LS, Branch WT, Cook CB, et al. Clinical inertia. Ann Intern Med. 2001;135(9):825-34. https://doi.org/10.7326/0003-4819-135-9200111060-00012.

26. Reach G, Pechtner V, Gentilella R, Corcos A, et al. Clinical inertia and its impact on treatment intensification in people with type 2 diabetes mellitus. Diabetes Metab. 2017;43(6):501-11. https://doi. org/10.1016/j.diabet.2017.06.003.

27. Sico IP, Oberle A, Thomas SM, et al. therapeutic inertia in prescribing biologics for patients with moderate-to-severe asthma: workshop summary. Patient Prefer Adher. 2021;7(15):705-12. https:// doi.org/10.2147/PPA.S303841.

28. Ulutaş F, Çobankara V, Şenol H, et al. A single center experience: Physician related diagnostic delay and demographic and clinical differences between patients with ankylosing spondylitis and non-radiographic axial spondyloarthritis. Rom J Intern Med. 2021. https://doi.org/10.2478/rjim2021-0004.

29. Steinmetz D, Tabenkin H. The 'difficult patient' as perceived by family physicians. Fam Pract. 2001;18: 495-500. https://academic.oup.com/fampra/ article/18/5/495/664879. Accessed 24 May 2021.

30. Smith S. Dealing with the difficult patient. Postgrad Med J. 1995;71:653-7. https://pmj.bmj.com/ content/71/841/653. Accessed 24 May 2021.

31. Hahn SR, Thompson KS, Wills TA, et al. The difficult doctor-patient relationship: somatization, personality and psychopathology. J Clin Epidemiol. 1994;47: 647-57. https://www.jclinepi.com/article/ 0895-4356(94)90212-7/pdf. Accessed 24 May 2021.

32. Smith S. Dealing with the 'difficult" patient.' In: Hind CRK, editor. Communication skills in medicine. London: BMJ Publishing Group; 1997. p. 101-14.

33. Hinchey SA, Jackson JL. A cohort study assessing difficult patient encounters in a walk-in primary care clinic, predictors and outcomes. J Gen Intern Med. 2011;26:588-94. https://doi.org/10.1007/ s11606-010-1620-6.

34. Hull SK, Broquet, K. How to manage difficult patient encounters. Fam Pract Manag. 2007. https://www.aafp.org/fpm/2007/0600/p30.html. Accessed 6 Aug 2019.

35. Cohen-Cole SA. Chapter 228 the "Difficult" medical patient. In: Walker HK, Hall WD, Hurst JW, editors. Clinical methods: the history, physical, and laboratory examinations. 3rd ed. Boston: Butterworths; 1990.

36. Martindale J, Goodacre L. The journey to diagnosis in AS/axial SpA: the impact of delay. Musculoskelet Care. 2014;12(4):221-31. https://doi.org/10.1002/ msc. 1080 .

37. Ogdie A, Benjamin Nowell W, et al. Real-world patient experience on the path to diagnosis of ankylosing spondylitis. Rheumatol Ther. 2019;6(2): 255-67. https://doi.org/10.1007/s40744-019-01537, https://www.ncbi.nlm.nih.gov/pmc/articles/ PMC6513959/. 